\title{
Fascial organisation of motor synergies: a hypothesis
}

\author{
Alessandro Garofolini (1), Daris Svanera (2) \\ (1) Institute for Health and Sport (IHES), Victoria University, Melbourne, Australia; \\ Independent researcher, Brescia, Italy \\ This article is distributed under the terms of the Creative Commons Attribution Noncommercial License (CC BY-NC 4.0) which permits \\ any noncommercial use, distribution, and reproduction in any medium, provided the original author(s) and source are credited.
}

\begin{abstract}
In the field of biomechanics and motor control understanding movement coordination is paramount. Motor synergies represent the coordination of neural and physical elements embedded in our bodies in order to optimize the solutions to motor problems. Although we are able to measure and quantify the movement made manifested, we do not have confidence in explaining the anatomical bases of its organisation at different levels. It is our contention that the flexible hierarchical organization of movement relies on the fascial structurers to create functional linkages at different levels, and this concept attunes with the neural control of synergies. At the base of movement organization there is a (somatic) equilibrium point that exists on the fascia where the neurologically- and mechanically-generated tensions dynamically balance out. This somatic equilibrium point is at the base of postural control, afferent flow of information to the nervous system about the state of the muscles, and of the coordinative preactivation of muscular contraction sequences specific for a synergy. Implications are discussed and suggestions for research and clinical applications are made.
\end{abstract}

Key Words: Motor control, degrees of freedom, myofascial continuity, movement coordination, muscle spindle.

The anatomical elements involved in the organisation of motor synergies, and voluntary movements in general, are still not clear for several reasons. First, the difficulty in explaining movement organisation which is inherited in the traditional anatomical understanding of the human body, often seen as the sum of the parts that compose it. ${ }^{1}$ Second, classical anatomy has been based for centuries on the concept that we can extract a part, we can study how it works by breaking it down to its components, and once we have all the parts extracted and analysed, the sum of all those parts will explain how the human body works. ${ }^{2}$ Although this view has been challenged over the years, ${ }^{3,4}$ our understanding of function is still biased by a segmental anatomical knowledge of our body. This is manifest in biomechanical models of the musculoskeletal system that represent muscles as independent units connected to the bones at their origin and insertion. ${ }^{5,6}$ In this perspective, muscle forces are transmitted serially, and the torque developed around a joint depends only on the muscle's torque arm geometrical configuration. Movement patterns are therefore, analysed through a linear framework of isolated muscle groups, based on singular muscle attachments and isolated joint actions. However, complex movements result from simultaneous interaction of multiple parts of many human systems and denying this may overlook the complexity of the human system and limit our understanding of movement organisation. An alternative view regards the human body as a tensegrity-like network, ${ }^{7}$ with the connective tissue (fascial structure) acting as linking component. ${ }^{8}$ In this view, the direct morphological continuum between muscles and fasciae is at the base of the mechanical interactions between agonist muscles (i.e., producing the same movement at a joint) as well as between antagonistic muscles $;^{9}$ this tensional continuous coordinates intermuscular and extramuscular force transmission. ${ }^{10}$ The central role of the fascia in movement coordination has been shown in a series of experiments, ${ }^{10-13}$ where muscle tendons were transposed (insertion shifted to antagonistic location) and movement recorded. The assumption that a muscle would change its action after tendon transposition was rejected: muscle still conserved its function due to the orientation of the connective tissue. This proves the controlling function of the fascia over muscle activity.

While evidence consistent with the role of synergies in movement organisation is growing, ${ }^{14-17}$ much work is still needed to delineate their anatomical basis. ${ }^{18}$ 
Although it is relatively easy to observe a synergistic movement, explaining the anatomical structures responsible for generating and organising such a movement is not an equally easy task. Research has focused on whether synergies have a neural origin, ${ }^{19}$ or they are a product of experimental or biomechanical constraints. ${ }^{20}$ It is believed that basic foundation of covariant muscle combinations (motor primitives) are modulated by the central nervous system to perform complex motor behaviors, ${ }^{21,22}$ that is, information is encoded into motor neurons (either by genetic design or learnt through experience) so that higher neural discharge rate is present in a preferred movement direction. ${ }^{23}$ However, by varying the length change of muscle groups individually, synergies between muscles emerge as a result of non-neural coupling, ${ }^{20}$ showing that the central nervous system does not need to control a group of muscles to observe muscle activation. ${ }^{24}$ Synergies are difficult to investigate because it is still not completely clear how the nervous system combines synergies, or how and where (anatomically) the synergies are scaled and weighted..$^{25}$ In our hypothesis, both neural and nonneural control of motor synergies coexist and they are linked through the fascial system. Aim of this review is to present possible anatomical explanations of the phenomena - synergies - described and quantified in motor control. We will look at synergies and their pillars from a fascia point of view, giving evidences for an alternative way of thinking about movement organisation. Before proceeding to examine synergies, it is important to define the fascial anatomical organisation, and its physiological meaning.

\section{Fascial System}

Fasciae are classified as a proper connective tissue that are dense and regular, ${ }^{26}$ made of collagen and elastic fibres; the former gives structure while the latter gives elasticity to the tissue. At different levels, the type of fibres and their orientation will define the role of the connective tissue. In this section we will illustrate the muscular fasciae (or deep fasciae); for a comprehensive review of the cellular characteristics of the fasciae see Stecco C, Macchi V, Porzionato A, et al., 2011 and Stecco C. 2014..$^{26,27}$ The muscular fascia (Figure 1) is divided in (from inner to outer layers):

1. The endomysial fascia surrounds muscle fibres; its collagen fibres are directly connected to the basal lamina overlying each muscle fibre. ${ }^{28}$ The motor (alpha axon) endplate terminates on the endomysium. The outer layer of the endomysium is made of loose connective tissue ensuring gliding between muscle fibres.

2. The perimysial fascia surrounds secondary bundles of muscles. With a small inner layer (connected to endomysial fascia) of loose connective tissue, an external layer of gliding, and an intermediate layer of

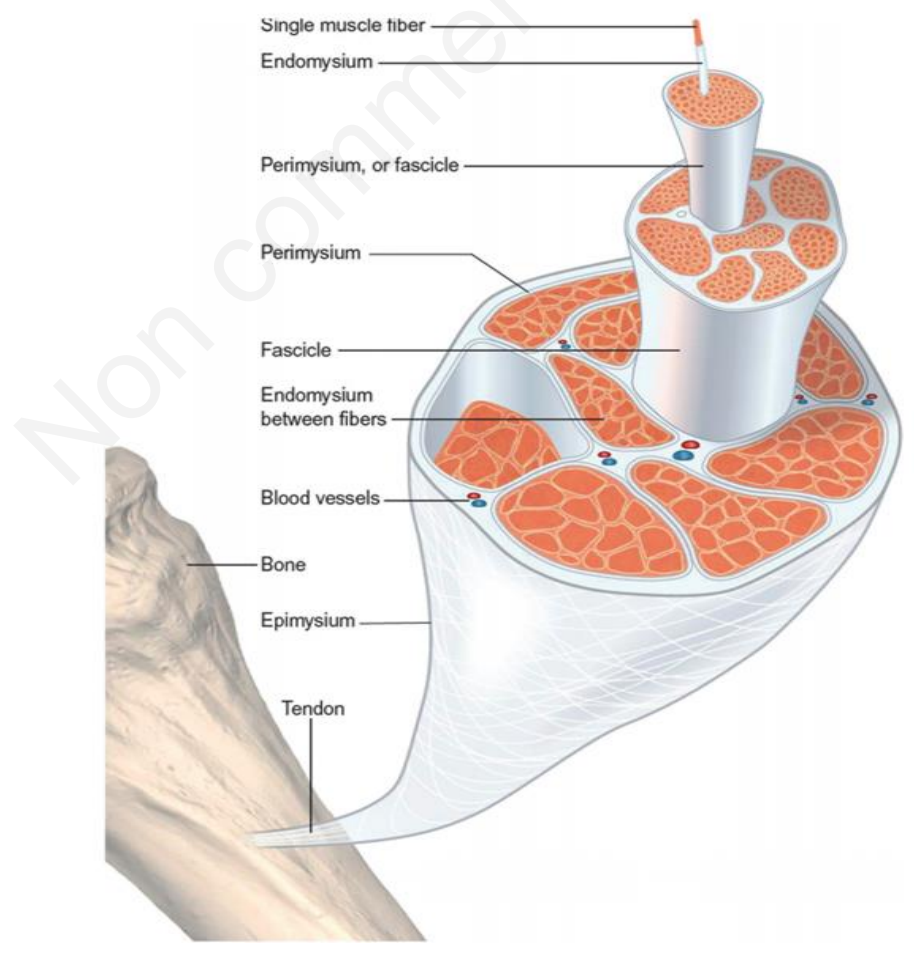

Fig 1. Representation of the muscular fasciae. Aponeurotic fascia is not displayed as it will contain all muscles with similar directional meaning.

Reproduced with permission from Handspring Publishing Ltd, taken from "Fascia: what it is and why it matters" by D. Lesondak (2018). 
collagen fibres, the perimysium has high resistance to traction, ${ }^{29}$ The ends of the neuromuscular spindle insert on to the perimysium.

3. The epimysial fascia surrounds the individual muscles (i.e. bicept brachi); it is a fibrous-elastic tissue closely connected to the muscle. Multiple septa exist connecting the epimysial fascia to the underlying muscle fibres and the perimysium, and the overlying aponeurotic fascia. The intermediate layer is constituted by about $20 \%$ of collagen fibres and elastic fibres; those give the ability to the epimysial fascia to resist tractions. ${ }^{29}$

4. The aponeurotic fascia is the outermost component of the muscular (deep) fascia. It is composed of two (or three) independent layers of about $1 \%$ elastic fibers and $80 \%$ collagen fibres arranged longitudinally, transversally, and obliquely. ${ }^{30}$ Each layer has parallel collagen fibres separated from the underlying one by a layer of loose connective tissue that allows collagen fibers to glide freely one onto the other. ${ }^{27}$ The function of the aponeurotic fascia is to transmit forces, and thanks to the different orientations of the collagen fibres, the aponeurotic fascia can transmit forces in any direction.

\section{Somatic Equilibrium Point (SEP)}

Only $70 \%$ of the extrafusal muscle fibres (those that lay outside the muscle spindle) have a tendinous insertion, while $30 \%$ have a fascial insertion, ${ }^{31}$ which allow muscle tension to be transmitted onto the fascia at the level of the epimysium. $^{29}$ Similarly, the intrafusal muscle fibres (those that lay inside the muscle spindle) tension the perimysium. Thanks to the bindings between different layers and their collagen nature (see previous section), a somatic point of equilibrium is formed on the epimysium that represent the equilibrium between alpha motorneurons' activity (extrafusal muscle fibres activation) and gamma motor-neurons' activity (intrafusal muscle fibres activation). We hypothesise this neuro-mechanical system to be the anatomical base for basal muscle tone and muscle synergies. We acknowledge that computational models already exists for alpha-gamma coordination, ${ }^{32}$ and they comprised physiologically realistic spinal circuitry, muscles, proprioceptors, and

\begin{tabular}{|c|c|c|c|c|c|}
\hline Movement intention & Premotor area & Action Plan & & 25 & \\
\hline \multirow{5}{*}{$\begin{array}{l}\text { Movement } \\
\text { organisation }\end{array}$} & $\begin{array}{c}\text { Primary motor area } \\
\text { Alpha \& Gamma neurons }\end{array}$ & $\begin{array}{c}\text { Action } \\
\text { destabilization (ASA) }\end{array}$ & Perimysium & Primary motorneurons - co-activation gamma-alfa & Synergy- 0 \\
\hline & $\begin{array}{c}\text { la \& lla } \\
\text { Alpha \& Gamma neurons }\end{array}$ & $\begin{array}{l}\text { Unidirectional } \\
\text { segmental } \\
\text { movement }\end{array}$ & Epimysium & $\underset{\text { Elemental variables - (Muscle and segmental joint) }}{\text { Task-1 (Regulation of Muscle activity }}$ & Synergy- 1 \\
\hline & Spinal cord & $\begin{array}{c}\text { Unidirectional global } \\
\text { movement }\end{array}$ & $\begin{array}{l}\text { Aponeurotic } \\
\text { fascia } \\
\text { Epimysium }\end{array}$ & 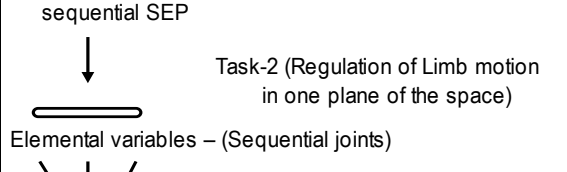 & Synergy- 2 \\
\hline & Spinal cord & $\begin{array}{r}\text { Bidirectional } \\
\text { movement }\end{array}$ & $\begin{array}{l}\text { Aponeurotic } \\
\text { fascia } \\
\text { Retinacula }\end{array}$ & $\begin{array}{l}\text { SEP in diagonal organisation } \\
\text { Task-3 (Regulation of Limbs motion } \\
\text { in two planes of the space) }\end{array}$ & Synergy- 3 \\
\hline & Spinal cord & $\begin{array}{c}\text { Multidirectional } \\
\text { movement }\end{array}$ & $\begin{array}{l}\text { Aponeurotic } \\
\text { fascia } \\
\text { Retinacula }\end{array}$ & $\begin{array}{l}\text { SEP in spiral organisation } \\
\text { in three planes of the space) } \\
\text { Elemental variables - (Limbs in spiral movements) }\end{array}$ & Synergy- 4 \\
\hline
\end{tabular}

Fig 2. Motor synergies characteristics. 
skeletal biomechanics. Although model behavior can match human movement and postural data, it omits the fascial system, thus its contribution to sensorimotor function.

\section{Synergies}

\section{Degrees of freedom}

How the body organises redundant degrees of freedom (DoF) is a crucial question in motor control. ${ }^{33}$ For a system to be redundant, a hierarchical organisation is assumed in which each level has more elemental variables (i.e. $n$ muscles) than the higher level (i.e. $m$ joints) where $\mathrm{m}<\mathrm{n}$. The other assumption governing redundant systems is that at any level, elemental variables are independent from one another. It is not surprising that this latter concept sounds reasonable; as we introduced before, it is a common thought that extracting an element from the system is not going to change the function of other elements in the system, therefore elements are assumed to be independent. However, there are many anatomical evidences that disprove this concept; for instance, muscles partially insert on the aponeurotic fascia, ${ }^{26,34}$ some muscles are biarticular (crossing two joints) or multi-articular (crossing multiple joints). ${ }^{27}$ Interconnections (at the same level, and between levels) creates interdependency hence the DoF are already reduced. Notice, only acknowledging that muscles insert onto the fascia changes dramatically how we see movement organisation. Not only muscles produce a pulling force on the bone, but they also stretch the fascia creating sequences of movements (discussed later). Therefore, DoF are reduced (or better, organized) anatomically through the fascial system, thus limiting the number of computations needed at any higher level (i.e. cerebral cortex). The principle of abundance, introduced later, ${ }^{15,35}$ is different from the problem of motor redundancies in the sense that movement is not constrained into a single solution, but rather the system uses all available DoF at lower levels to facilitate groups of solutions equally able to solve the task-at-hand. Solutions emerge based on the actual state of the body (anatomical constraints), environmental constraints, and task constraints. ${ }^{36}$ The fascial system anatomically explains how 'movement selection' is achieved at lower levels of the chain making the intention possible: the focus of the person doing the movement is on the final effectors (in voluntary movement initiation) or on control parameters (in continuous movements), while the focus of the body is on producing the movements needed to meet the request. Like the horses pulling the chariot are coordinated by the charioteer that in turn, works to meet the passenger's desire.

\section{Motor Synergy}

Although many definitions of motor synergies may be found in literature, motor synergies are best described as the hypothetical neural mechanism that ensure taskspecific covariation of elemental variables providing for desired stability properties of an important (performance) variable. In the act of reaching to an object, performance variable is the position of the hand in space, while the elemental variables are the arm's joints angles, and torque resulting from muscle fibres contraction. Functionally, elemental variables co-vary to stabilize the performance variable. This can be also extended to posture stabilization. Multiple synergies can arise at the same time to solve multiple problems: imagine you are walking on the street and you want to check the time. Multiple synergies are involved in this rather simple task; first, walking is controlled and organized passively by feedback loops (continuous movement). Then, the intention of looking at the watch activates the unconscious anticipatory synergy adjustments, ${ }^{14,37}$ which work to destabilize the synergetic movement of the arm swing and to change the muscle fibre threshold to a new somatic equilibrium point (see below). As we consciously start the arm movement, two different synergies (different tasks) for the two arms arise, and this has an influence on the 'background' synergy of walking that now needs to compensate for the ceased arm swing. The function of the fascial system is to automatically help the person (self-organisation); the details of the operation would distract the walker from his task of keep walking at the same speed, in the same direction. Even though feedbacks information may reach the nervous system, this information is only relevant to the functioning of the subservient system, and it should remain private to that mechanism. ${ }^{38}$

\section{Integration with the fascial system}

Figure 2 represents the hypothesized hierarchical motor synergy organisation integrating the fascial system. Synergistic levels are organized in a nesting of interconnected soft tissues forming physical and functional linkages that ensure continuity between muscles and connective tissue. Lower level synergies are building blocks which higher level synergies are based upon. The first synergistic level 'synergy-0' concerns organisation and coordination of muscle spindles with a feedforward (not only feedback) action of converging spindles' tractional forces onto the fascia. The proposed premise is that alpha and gamma neurons are always active, even though their activation level is under threshold, electric potential is keeping tension on the muscle fibres, both intra- and extrafusal. This explains the basal muscle tone. When we relax a muscle, some underlying tension is still perceivable. In the case of voluntary (pre-planned) actions, primary motor area activates, through primary motor neurons, both alpha and gamma motor neurons; this co-activation regards groups of motor neurons with the same functional direction. That is, all the motor neurons that activates motor units that produce movement in the same direction. Alpha preactivation is essential to decrease their gain (activation threshold; at the same time, because gammas have a smaller size, they reach activation threshold before the 
alphas causing a change in tension on the somatic equilibrium point (SEP) through muscle spindles action and their insertion on the perimysium (see below). It is important to notice that at this level, no apparent (detectible) movement is present. Synergy-1 organises motor units (belonging to the same segment) that have the same direction of contraction, and the segment as a whole for movements executed on a single plane (i.e. sagittal, frontal, or transverse) - unidirectional segmental movements. Synergy-1 has its bases on the physical emergency of the SEPs: multiple muscle spindles are organized functionally through the SEPs and if the fascia is elastic, the intrafusal fibres of the muscle spindles are able to shorten, sending efferent signals (Ia and IIa) to alpha motor neurons with the same directional meaning. The activation of the alpha motor neurons produces the contraction of the extrafusal fibres (muscle gross contraction). Motor units are therefore pre-activated and pre-informed about the position of muscles and joints thanks to the perceptive function of the SEPs. In synergy1 we talk about segmental SEP as the converging point of the resultant force developed by the muscle spindles that are functionally linked to the alpha motor neurons ipso-directional in a segment. The advantage of having segmental SEPs is that the nervous system does not receive afferent information from single muscle spindle of a muscle, but rather it gathers information from specific points - SEPs - that exist thanks to the $30 \%$ insertion of the extrafusal muscle fibers onto the fascia. Moreover, the segmental SEP is physically specular (equal direction, opposite verse) to the force developed

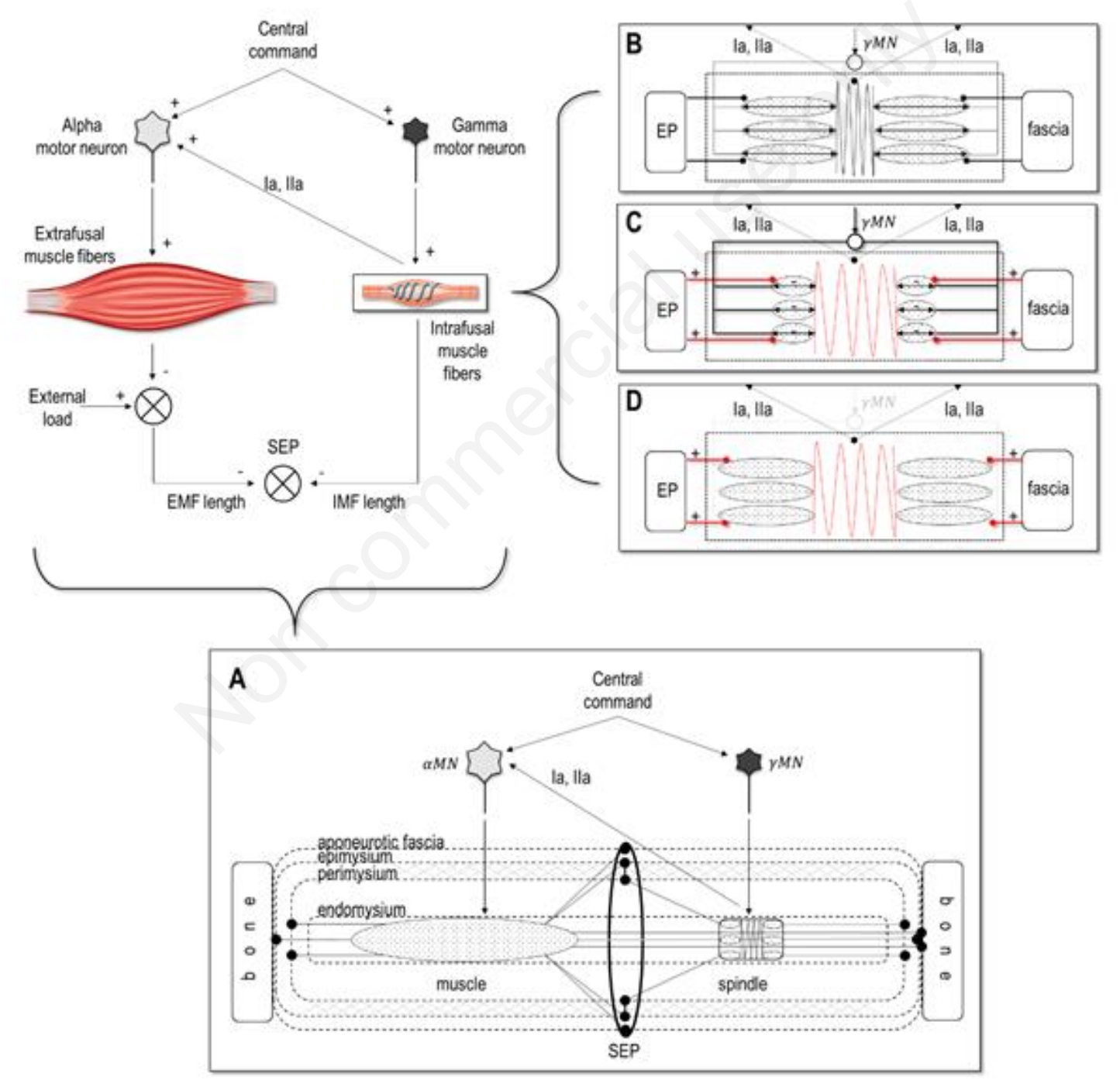

Fig 3. Schematic of the somatic equilibrium point (SEP) between extrafusal and intrafusal muscle fibres. (A) Fascial relation with muscle spindle and the muscle. The spindle is represented as an extensive spring $(B)$ that can be compressed by neural activation $(C)$, or by tensional forces $(D)$. Motor synergies characteristics. 


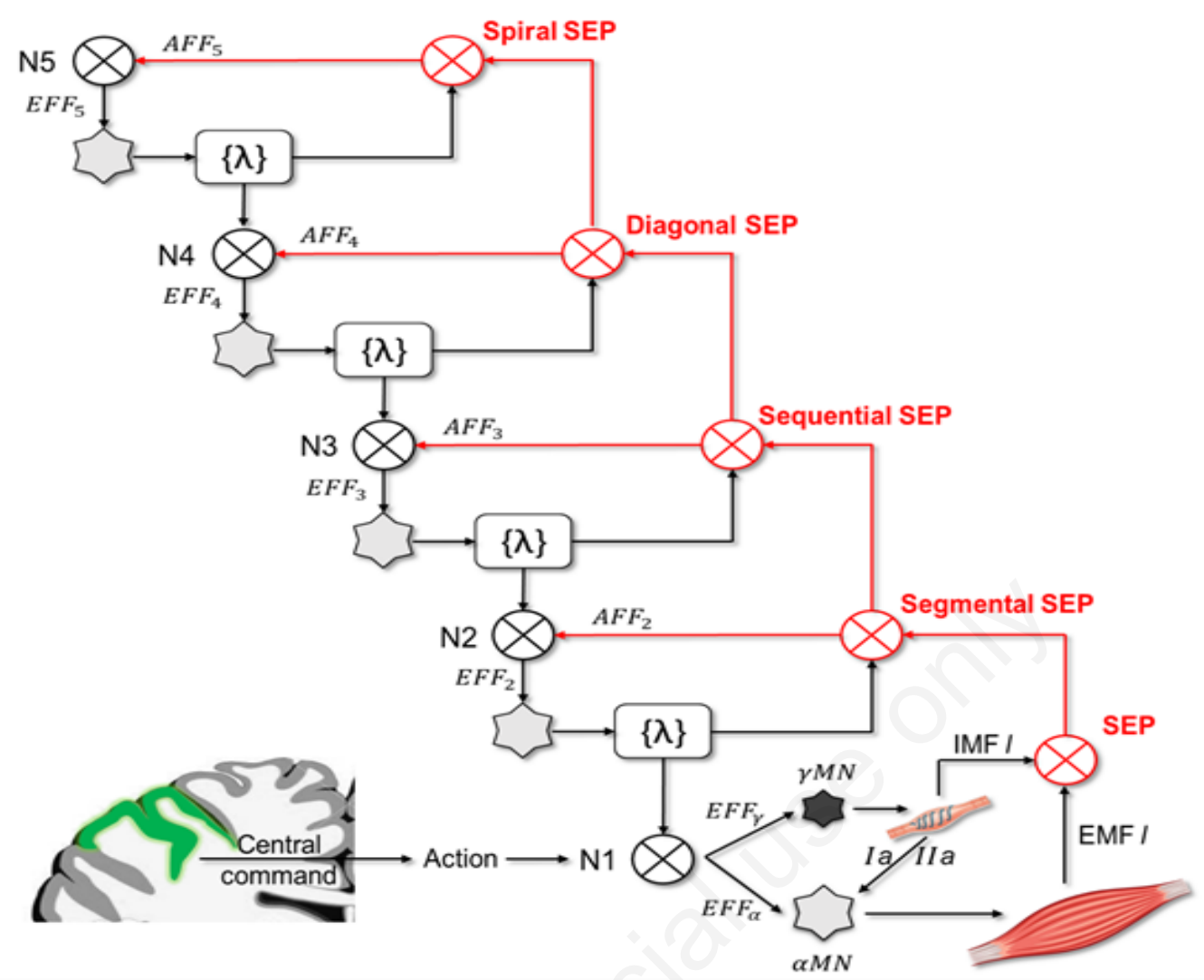

Fig 4. Hierarchical representation of motor synergies based on the hypothesized integrated neuro-mechanical model. $A F F=$ afferent; $E F F=$ efferent; $S E P=$ somatic equilibrium point; $N \#=$ group of neurons; $I M F l=$ intrafusal muscle fibers length; $E M F l=$ extrafusal muscle fibers length; $M N=$ motor-neuron; $\lambda=$ muscles length.

by the extrafusal fibres on the tendon pulling the bone (gross movement). Synergy-2 interests the coordination of multiple joints of a limb (or the trunk) anatomically and functionally linked through sequential SEP in what we call unidirectional global movements. As we will see, SEPs lay onto the epimysium but the epimysium is linked, through septa, to the aponeurotic fascia. Therefore, any traction on a segmental SEP will be transferred to SEPs of adjacent segments, and because the aponeurotic fascia is seamless, all SEPs ipsodirectional are functionally connected. Thus, motor units of adjacent segments are activated (myotatic stretch reflex) by passive stretch of muscle spindles connected to the SEP of that segment. Synergy-3 organises motor units, joints, and spinal reflexes involved in bidirectional movements classified as segmental motor schemes (for shifting of joints from one plane to another), or global motor schemes (for shifting of a limb or the trunk onto intermediate planes - diagonals). Synergy-3 deals with everyday movements, those that are organized on an infinite number of planes. At peripheral level, multiple segmental SEPs converge in a somatic equilibrium point at a higher synergic level, located where the fascial laminae molten: retinacula. This hierarchically higher SEP coordinates, through its tension (and a proprioceptive feedback) segmental SEPs involved in movements on intermediate planes. Higher SEPs are organized into diagonals to regulate segmental (or global) movement of a limb (or the trunk) in motor schemes. Synergy-4 regulates complex movements involving multiple joints and multiple planes classified as segmental or global multi-directional movements (fine or complex motor gests respectively). This last synergistic level is governed by SEPs anatomically and functionally linked in a spiral way in order to organise movements executed onto the three planes. Synergy-4 organises fine gests like piano playing and global movements such as soccer kick or volley strike. To sum up, an ongoing tension on the fascia is present due to alpha and gamma under-threshold activity (basal muscle tone); following a neural (gamma) stimuli, muscle spindles stretch the epimysial fascia of a muscle before the contraction of the muscle at its origin and insertion. The muscle spindles activated by the same gamma impulse (impulse that is assumed to have the same directional meaning) generate fascial tractions that will 
converge in a point onto the fascia we called somatic equilibrium point. In this view, the SEP represents (i) the somatic point of equilibrium between the fascial tension of the intrafusal and extrafusal muscle fibers; and (ii) the point of application of the vectorial resultant of all those fascial tractions; in response to which, the muscle belly deforms itself in the three planes of space simultaneously, changing volume and form of the belly before shortening longitudinally. The SEP is at the base of three actions: the postural destabilisations, the afferent flow of information to the nervous system about the state of the muscles, and the coordinative pre-activation of muscular contraction sequences specific for a synergy. Now that we have established the importance of the fascial system in movement organisation, in the next section we will describe the structure and function of what we hypnotize be the fundamental unit that organises movement at the lowest

\section{The muscle spindle}

Traditionally seen as a feedback regulator system (stretch sensitive organ) ${ }^{39}$ the muscle spindle has gained its importance as a feedforward element in recent years . ${ }^{29,40-}$ ${ }^{43}$ We hypothesize the muscle spindles are designed to regulate muscle activity and movement initiation (for voluntary actions), and thanks to their embedment into the epimysial fascia they underpin synergies. The role of the muscle spindles has been compared with the servocontrol of the road wheels in a car. ${ }^{44}$ In brief, a sensor detects the difference between the position of the steering wheel (corresponding to the position of the road wheels that the driver wants them - road wheels - to be) and the actual position of the road wheels. The system works to minimize the difference between the two: actual position and desired position of the road wheel. Similarly, we hypothesise a double role of the muscle spindle: it modulates $\alpha$ activation (through Ia and IIa afferent) while also tensioning the epimysial fascia. Thanks to the shared connections with the fascia, the tension (tone) of the extrafusal fibres (i.e. muscle) is also modulated (Figure 3 ). Figure $3 \mathrm{~A}$ depicts the hypothetical neural and physical connections between spindles and muscle fibres. Notice that the two points of anchorage for the muscle fibres are not both on the tendon but rather one (the SEP) is "somewhere" on the belly of the muscle. The position of the SEP will be different for movements on different planes. Our hypothesis is that spindles have a basal tension due to under-threshold activation (Figure 3B) that act as compressive spring when their intrafusal fibres are neurologically activated through gamma motor neurons (Figure 3C); while they are stretched when a physical force is applied to them (through the fascia) by extrafusal fibres (Figure 3D). Muscle fibres within the muscle spindles (intrafusal) are innervated by the gamma $(\gamma)$ motor-neurons while muscle fibres within the muscle (extrafusal) are innervated by the alpha $(\alpha)$ motorneurons. The concomitant descending innervation for $\gamma$ and $\alpha$ motor-neurons is usually under threshold for alpha motor neurons while gamma are activated. We hypothesise that this pre-activation, (called anticipatory synergy adjustments) ${ }^{14}$ is necessary to tune in the muscles with the task to be performed. The anticipatory synergy adjustments activates the muscle spindles (of muscles that will be involved in the future movement of the end-effector) adjusting their intrafusal fibres length; once the voluntary impulse activate the extrafusal fibres they will already know the level of activation required (feedforward control). The performance variable would not change until voluntary activation is started, thus, synergy destabilisation is an automatic, involuntary action representing a healthy change in basal muscle tone. ${ }^{45,46}$ The equilibrium-point hypothesis, ${ }^{47,48}$ and more recently the referent configuration hypotheses, ${ }^{14,49}$ describe closely the function of the anticipatory synergy adjustments and the role of the spindles. However, what has been described functionally (and analytically), has not had anatomical support. The parameters of feedbackbased mechanisms at each level of the hierarchy that control lower levels in a feedforward manner have been described as having unknown physiology. Although this sensory receptors were assumed to have a central role in motor synergy (and movement organisation), they were located with uncertainty in remote muscles within the various segments of the body. We are here presenting an anatomical framework explaining the organisation of the fascial system that runs in parallel to the neurological control of synergies while modulating its output (Figure 4). The central command defines a referent configuration (muscles length) for the body that activates a group of neurons (N1) based on subthreshold depolarisation and minimal excitatory afferent input (AFF), thus leading to $\mathrm{N} 1$ response (EFF). ${ }^{50}$ At each hierarchical level, a reference configuration exists, hence each level represents a feedback system controlled in a feedforward way. We argue that while the command goes to the end effector, the synergetic movements are controlled from the lowest level up by transfer of tension through the fascial system: the tension at the SEPs defines the somatic equilibrium point for muscles of the end effector; in turn, the tension on the segmental SEP defines the somatic equilibrium point for the segmental joints interested by the movement; the tension on the sequential SEP defines somatic equilibrium point for the sequential joints (limb motion on one special plane); tension on the diagonal SEP defines the somatic equilibrium point for limbs in diagonal movements (motion in two planes); and finally, tension on spiral SEP defines the somatic equilibrium point for limbs in spiral movements (limbs motion on multiple planes of the space). These anatomical (fascial) sensors act as bridges between different synergistic levels; they allow transfer of tension from the lowest level to the highest, ensuring proper muscle activation hence proper movement. Their organisation is still redundant (\# of SEPs $<$ \# of segmental SEPs < \# of sequential SEPs) and automatic (selforganisation), but they act in an opposite direction than 
the neural control. It is important to highlight that not all synergic levels will be active for any given movement. In fact, activation of multiple level synergies happens in response to the complexity of the movement and on the number of body segments involved in the action. Also, the type of movement will define synergies: i.e. for 'rapid pre-planned learned movement' the anticipatory synergy adjustments and its preparatory effects will be present; however, for rapid but 'unintended naive movements' activation of alpha motor neurons will overwrite the gamma pathway, hence, movement will be less controlled and more prone to injuries as the anatomical structures linked with extrafusal fibres are not able to react as quickly to those muscle contractions. On the other hand, 'cyclic movements' rely heavily on fascial control and organisation of movements so that we do not need to worry about it and more important we can accomplish multiple tasks together.

\section{Research Implications}

Muscle modelling has been introduced to understand muscle contribution to movement: by knowing body's segments length, their kinematics (segments' configuration) and kinetics (force produced on the ground), it is possible to estimate muscle groups moment of force (i.e. extensor moment); from here, based on anatomical knowledge of origin and insertion of muscles, it is possible to estimate their contribution. ${ }^{51}$ However, if we consider the fascia creates anatomical links between muscles and groups of muscles, ${ }^{34}$ and if we recognise that the insertion and origin of the, ${ }^{52}$ the foundation of modelling shakes. Away from suggesting modelling is not worth pursuit, we echo the concerns recently brought about on the accuracy of modelling estimation. ${ }^{53}$ hence clinical inferences based on modelling may be pushing the boundaries of how much we can estimate from a 'simple' model and what clinical relevance it has. Robotics may greatly improve by introducing a pseudofascial system into development of assistive devices: for instance, for active and passive exoskeletons, the distribution of forces along the body segments has been always the ultimate challenge. Feedforward control based on feedback information at a local level could be improved by introducing interlinks between levels so that all elements act in harmony with the all system. The fascial system presupposes a spiral distribution of the somatic equilibrium points on the body, thus forces are not distributing linearly through the body segments and through the anatomical structures. Perhaps by mimicking fascial organisation robotic models may be more successful in assisting people in need.

\section{Clinical Implications}

In case of traumatic events, structural overloading, metabolic disorders, or clinical surgeries the fascial system may lose its functional properties hence affecting the control of movement. Rehabilitative intervention may integrate their focus on muscle health with connective tissue health. Working on the anatomical receptors (somatic equilibrium points) will re-establish a correct activation of the subsequent (hierarchically lower) components. For instance, for sport skills involving spiral movement would require an intervention on SEP laying on the movement sequence interested. Not only may those point be used for treatment but also for evaluation. ${ }^{54}$ In fact, if these point represent somatic equilibrium points, they reflect the balance between muscle force and fascial tension: in a healthy body the fascial system is free to move and transmit tension along the path, however, in case of rigidity of the fascial structures, elements will not be in balance resulting in pain and possibly leading to injuries. ${ }^{55}$

\section{Hypothesis testing}

Our hypothesis can be tested using either a computational model or in-vivo measurements. The former, by implementing the fascial system (with all its layers), will extend the alpha-gamma model. ${ }^{32,56}$ Such model will demonstrate the coordinative role of the fascial system over posture maintenance and movement coordination. However, the approach presumes knowledge of the elastic properties of each layer, and of the link type between different layers. The latter comprises quantum dot based flexible strain sensors,${ }^{57}$ to directly measure the stretch of the fascia caused by a muscle contraction. Photo-luminescence changes when strain is applied. This minimally-invasive technique may be applied over the flexible strain sensors made of polymers firmly attached to the muscle fascia. If the SEP exists, and therefore our hypothesis is valid, we may be able to measure points of high stress on the fascia where the forces are converging during controlled movements.

\section{Conclusion}

Many researchers and scientists have described and quantified movement organisation from a mathematical and physiological point of view. With this essay we corroborate the ideas presented in the equilibrium point hypothesis and reference configuration hypothesis, adding a more in-depth anatomical explanation for movement organisation. Implementing the fascial system in those theories gives a more complete view on how human body organizes movement. ${ }^{58} \mathrm{We}$ elucidated the communality between these theories and suggested possible research and clinical applications of the concepts herein discussed. We may be still far away from fully understanding how human body works, but including the connective tissue in motor control may enlighten some of the anachronistic concepts that have lead the scene of motor control for many years.

\section{List of acronyms}

AFF - minimal excitatory afferent input

DoF - degrees of freedom

EFF - N1 response

SEP - Somatic Equilibrium Point 


\section{Authors contributions}

Authors equally contributed to the conceptualization, drafting and writing of the manuscript.

\section{Acknowledgments}

We thanks Dr Simon Taylor for comments that greatly improved the manuscript. Funding This work was supported by the Fascial Manipulation Institute, via Cavalieri di Vittorio Veneto, 19, 35129 Padova (Italy)

\section{Conflict of Interest}

The authors declare no conflict of interest.

\section{Ethical Publication Statement}

We confirm that we have read the Journal's position on issues involved in ethical publication and affirm that this report is consistent with those guidelines.

\section{Corresponding Author}

Alessandro Garofolini. Institute for Health and Sport (IHES), Victoria University, Melbourne, Australia. ORCID: 0000-0002-1789-2362 Phone: 0401613287 Email: alessandro.garofolini1 @live.vu.edu.au

\section{E-mail of co-author}

\section{Daris Svanera: sva8@hotmail.it}

\section{References}

1. Wand BM, O'Connell N. German acupuncture trials for chronic low back pain. Arch Intern Med 2008;168:1011; autor reply2.

2. Laplace PS. A philosophical essay on probabilities, translated into english from the original french 6th ed. By truscott, fw and emory, fl. Dover Publications, New York; 1951.

3. Andersen H. The history of reductionism versus holistic approaches to scientific research. Endeavour 2001;25:153-6.

4. Lidicker WZ. The synergistic effects of reductionist and holistic approaches in animal ecology. Oikos 1988;53:278-81

5. Arnold EM, Delp SL. Fibre operating lengths of human lower limb muscles during walking. Philos Trans R Soc Lond B Biol Sci 2011;366:1530-9.

6. Arnold EM, Hamner SR, Seth A, et al. How muscle fiber lengths and velocities affect muscle force generation as humans walk and run at different speeds. J Exp Biol 2013;216:2150-60.

7. Levin SM, Martin D-C. Biotensegrity. In: Schleip R, Findley TW, Chaitow L, Huijing PA, editors. Fascia: The tensional network of the human body. Oxford: Churchill Livingstone; 2012. p. 137-42.

8. Schleip R, Jager H, Klingler W. What is 'fascia'? A review of different nomenclatures. J Bodyw Mov Ther 2012;16:496-502.

9. Huijing PA. Epimuscular myofascial force transmission between antagonistic and synergistic muscles can explain movement limitation in spastic paresis. J Electromyogr Kinesiol 2007;17:708-24.
10. Maas H, Sandercock TG. Force transmission between synergistic skeletal muscles through connective tissue linkages. Journal of Biomedicine and Biotechnology 2010;2010.

11. Riewald SA, Delp SL. The action of the rectus femoris muscle following distal tendon transfer: Does it generate knee flexion moment? Dev Med Child Neurol 1997;39:99-105.

12. Maas H, Huijing PA. Mechanical effect of rat flexor carpi ulnaris muscle after tendon transfer: Does it generate a wrist extension moment? J Appl Physiol 2011;112:607-14.

13. Maas H, Huijing PA. Myofascial force transmission between transferred rat flexor carpi ulnaris muscle and former synergistic palmaris longus muscle. Muscles Ligaments Tendons J 2011;1:127.

14. Latash ML, Zatsiorsky V. Biomechanics and motor control: Defining central concepts: Academic Press; 2015.

15. Latash ML. The bliss (not the problem) of motor abundance (not redundancy). Exp Brain Res 2012;217:1-5

16. Latash ML, Gorniak S, Zatsiorsky VM. Hierarchies of synergies in human movements. Kinesiology (Zagreb) 2008;40:29-38.

17. Latash ML, Scholz JP, Schoner G. Toward a new theory of motor synergies. Motor Control 2007; 11:276-308.

18. Profeta VLS, Turvey MT. Bernstein's levels of movement construction: A contemporary perspective. Hum Mov Sci 2018;57:111-33.

19. Bizzi E, Cheung VC. The neural origin of muscle synergies. Front Comput Neurosci 2013;7:51.

20. Kutch JJ, Valero-Cuevas FJ. Challenges and new approaches to proving the existence of muscle synergies of neural origin. PLoS Comput Biol 2012;8:e1002434.

21. Bizzi E, Mussa-Ivaldi FA, Giszter S. Computations underlying the execution of movement: A biological perspective. Science 1991;253:287-91.

22. Ivanenko YP, Grasso R, Zago M, et al. Temporal components of the motor patterns expressed by the human spinal cord reflect foot kinematics. Journal of neurophysiology 2003;90:3555-65.

23. Cherian A, Fernandes HL, Miller LE. Primary motor cortical discharge during force field adaptation reflects muscle-like dynamics. Journal of neurophysiology 2013;110:768-83.

24. De Groote F, Jonkers I, Duysens J. Task constraints and minimization of muscle effort result in a small number of muscle synergies during gait. Frontiers in computational neuroscience 2014;8:115.

25. Ivanenko YP, Cappellini G, Dominici N, et al. Modular control of limb movements during human locomotion. J Neurosci 2007;27:11149-61.

26. Stecco C, Macchi V, Porzionato A, et al. The fascia: The forgotten structure. Italian journal of anatomy 
and embryology $=$ Archivio italiano di anatomia ed embriologia 2011;116:127-38.

27. Stecco C. Functional atlas of the human fascial system e-book: Elsevier Health Sciences; 2014.

28. Gray H, Standring S. Gray's anatomy: The anatomical basis of clinical practice: Churchill Livingstone; 2008.

29. Stecco L. Atlas of physiology of the muscular fascia: Piccin; 2016.

30. Stecco C, Pavan PG, Porzionato A, et al. Mechanics of crural fascia: From anatomy to constitutive modelling. Surgical and radiologic anatomy : SRA 2009;31:523-9.

31. Patel TJ, Lieber RL. Force transmission in skeletal muscle: From actomyosin to external tendons. Exerc Sport Sci Rev 1997;25:321-63.

32. Li S, Zhuang C, Hao M, et al. Coordinated alpha and gamma control of muscles and spindles in movement and posture. Frontiers in computational neuroscience 2015;9:122.

33. Bernstein NA. The co-ordination and regulation of movements. 1967.

34. Turrina A, Martinez-Gonzalez MA, Stecco C. The muscular force transmission system: Role of the intramuscular connective tissue. J Bodyw Mov Ther 2013;17:95-102.

35. Gelfand IM, Latash ML. On the problem of adequate language in motor control. Motor Control 1998;2:306-13.

36. Schoner G, Kelso JA. Dynamic pattern generation in behavioral and neural systems. Science 1988;239:1513-20.

37. Olafsdottir H, Yoshida N, Zatsiorsky VM, et al. Anticipatory covariation of finger forces during self-paced and reaction time force production. Neurosci Lett 2005;381:92-6.

38. Merton P. How we control the contraction of out muscles. Scientific American 1972;226:30-7.

39. Sherrington CS. On the anatomical constitution of nerves of skeletal muscles; with remarks on recurrent fibres in the ventral spinal nerve-root. J Physiol 1894;17:210 2-58.

40. Stecco L, Stecco C. Fascial manipulation. Fascia: The Tensional Network of the Human Body-EBook: The science and clinical applications in manual and movement therapy, Elsevier Health Sciences, 2013:1-335.

41. Kröger S. Proprioception 2.0: Novel functions for muscle spindles. Current opinion in neurology 2018;31:592-8.

42. Macefield VG, Knellwolf TP. Functional properties of human muscle spindles. Journal of neurophysiology 2018;120:452-67.

43. Giuriati W, Ravara B, Porzionato A, et al. Giuriati W, Ravara B, Porzionato A, et al. Muscle spindles of the rat sternomastoid muscle. Eur J Transl Myol. 2018;28:7904.
44. Merton PA. The silent period in a muscle of the human hand. J Physiol 1951;114:183-98.

45. Park $\mathrm{J}, \mathrm{Wu} \mathrm{YH}$, Lewis $\mathrm{MM}$, et al. Changes in multifinger interaction and coordination in parkinson's disease. J Neurophysiol 2012;108:91524.

46. Klous M, Mikulic P, Latash ML. Two aspects of feedforward postural control: Anticipatory postural adjustments and anticipatory synergy adjustments. J Neurophysiol 2011;105:2275-88.

47. Feldman AG, Levin MF. The equilibrium-point hypothesis-past, present and future. Progress in motor control: Springer; 2009. p. 699-726.

48. Feldman AG. Once more on the equilibrium-point hypothesis (lambda model) for motor control. J Mot Behav 1986;18:17-54.

49. Latash ML. Motor synergies and the equilibriumpoint hypothesis. Motor control 2010;14:294-322.

50. Danion F, Latash M. Motor control: Theories, experiments, and applications: Oxford University Press; 2010.

51. Horsman MDK, Koopman HFJM, van der Helm FCT, et al. Morphological muscle and joint parameters for musculoskeletal modelling of the lower extremity. Clin Biomech (Bristol, Avon) 2007;22:239-47.

52. Stecco C, Gagey O, Macchi V, et al. Tendinous muscular insertions onto the deep fascia of the upper limb. First part: Anatomical study. Morphologie 2007;91:29-37.

53. Schellenberg F, Taylor WR, Trepczynski A, et al. Evaluation of the accuracy of musculoskeletal simulation during squats by means of instrumented knee prostheses. Med Eng Phys 2018;61:95-9.

54. Stecco C, Macchi V, Porzionato A, et al. The ankle retinacula: Morphological evidence of the proprioceptive role of the fascial system. Cells Tissues Organs 2010;192:200-10.

55. Stecco C, Stern R, Porzionato A, et al. Hyaluronan within fascia in the etiology of myofascial pain. Surg Radiol Anat 2011;33:891-6.

56. Hao M, He X, Xiao Q, et al. Corticomuscular transmission of tremor signals by propriospinal neurons in parkinson's disease. PloS one 2013;8:e79829.

57. Chiang $\mathrm{C}-\mathrm{W}$, Haider $\mathrm{G}$, Tan W-C, et al. Highly stretchable and sensitive photodetectors based on hybrid graphene and graphene quantum dots. ACS applied materials \& interfaces 2016;8:466-71.

58. Lesondak D. Fascia: What it is and why it matters. Co-Kinetic Journal 2018; 75.

Submission: May 29, 2019

Revision received: August 01, 2019

Acceptance: August 02, 2019 